\title{
Review
}

\section{Cooperation between the default mode network and the frontal-parietal network in the production of an internal train of thought}

\author{
Jonathan Smallwood ${ }^{a, *}$, Kevin Brown ${ }^{b}$, Ben Baird ${ }^{c}$, Jonathan W. Schooler ${ }^{c}$ \\ a Department of Social Neuroscience, Max Plank Institute for Human Cognitive and Brain Sciences, Leipzig, Germany \\ ${ }^{\mathrm{b}}$ Department of Physics, University of California, Santa Barbara, CA, USA \\ ${ }^{\mathrm{C}}$ Department of Psychology, University of California, Santa Barbara, CA, USA
}

\section{A R T I C L E I N F O}

Article history:

Accepted 30 March 2011

Available online 3 April 2011

Keywords:

Task unrelated thought

Stimulus independent thought

Default mode network

Frontal-parietal network

Global workspace

Mind-wandering

\begin{abstract}
A B S T R A C T
The ability to generate and sustain an internal train of thought unrelated to external reality frees an agent from the constraints of only acting on immediate, environmentally triggered events. The current paper proposes that such thought is produced through cooperation between autobiographical information provided by the default mode network and a frontalparietal control network which helps sustain and buffer internal trains of thought against disruption by the external world. This hypothesis explains at least two features of the literature on internally guided thought. First, access to the top-down control system is a generally accepted prerequisite of conscious experience; this explains why activation of this system and default mode activity is often observed together during periods of internally guided thought. Second, because the top-down attentional control system has a limited capacity, internally and externally driven streams can come into conflict, with the result that perceptual information must be denied attentional amplification if the internal stream is to be maintained. This explains why internal thought is routinely associated with a state of perceptual decoupling, reflected in both measured anticorrelations between the default mode network and sensory areas and the manner in which task unrelated thoughts compromise task performance. This paper offers a hypothesis that should help to constrain and guide interpretations, investigations, and analyses of the neural processes involved in internally driven cognition. This article is part of a Special Issue entitled Special Issue The Cognitive Neuroscience.
\end{abstract}

(c) 2011 Elsevier B.V. All rights reserved.

\section{Contents}

1. Integrating top-down control and the default mode network (DMN): a global workspace model of internally guided

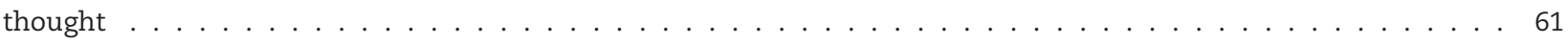

1.1. Prediction 1: conscious internal thought involves cooperation between elements of the DMN and the FPN. . 65

1.2. Prediction 2: engaging the internal stream of thought dampens sensory processing . . . . . . . . . . . 67

\footnotetext{
* Corresponding author. Fax: +49 34199402448.

E-mail address: smallwood@cbs.mpg.de (J. Smallwood).
} 


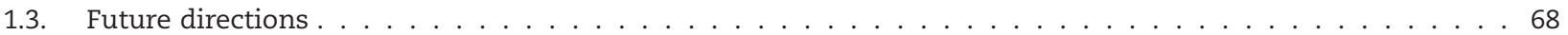

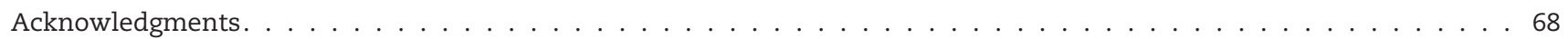

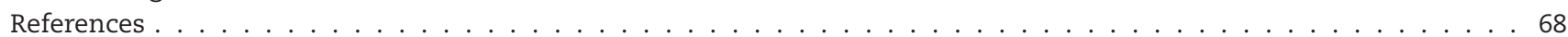

Whether lying on the beach and daydreaming about your next vacation, preparing for an upcoming meeting while showering, or solving a Sudoku puzzle on the train to work, consciousness often neglects immediate perceptual input to focus on an internal train of thought. The significance of this was not lost on William James when he suggested his well-known definition of attention: "Everyone knows what attention is. It is the taking possession by the mind, in clear and vivid form, of one out of what seem several simultaneously possible objects or trains of thought" (James, 1890, p. 403-404, emphasis added). Experience sampling studies confirm that internal trains of thought make up a significant proportion of cognition, with estimates suggesting that as much as $30 \%$ of a person's time is engaged internally (Smallwood and Schooler, 2006; Kane et al., 2007). Despite this fact, cognitive neuroscience research in the last century has largely focused on expanding our understanding of how externally oriented attention operates (Corbetta and Shulman, 2002; Posner and Petersen, 1990).

In the last decade, however, our understanding of how attention bears on internally generated thoughts has seen rapid growth, largely because of a puzzling observation from cognitive neuroscience that a coordinated system of brain regions, later dubbed the 'default mode network' (DMN, Raichle et al., 2001) shows increased activity when individuals have no demanding perceptual task to perform. The core brain areas of this network include the ventral medial pre-frontal cortex (vMPFC), the posterior cingulate cortex (PCC), the inferior parietal lobule (IPL), the dorsal medial prefrontal cortex (dMPFC) and the hippocampal formation (HF) (for a review see Buckner et al., 2008). Buckner and colleagues have suggested that the DMN is a coherent system which includes two main hubs: i) the PCC, and ii) the MPFC. The dMPFC and HF are also both correlated with other regions of the DMN; it is now thought that the HF and dMPFC are two distinct subsystems that are connected to the two hubs of the larger DMN.

Despite general agreement that the DMN is associated with stimulus independent cognition (Buckner et al., 2008), debate continues on the specific role the DMN plays. Can extensive DMN activity be attributed solely to spontaneous thought or is the activation too broad for such a purpose (Raichle, 2009; Raichle and Snyder, 2007)? Does the DMN aid in the generation of internal trains of thought or is this merely an epiphenomenal feature of a system that provides vigilance during lapses of external attention (Gilbert et al., 2007)? Are default processes functional, serving operations such as autobiographical planning (Smallwood et al., 2009, 2011; Smallwood, 2010; Spreng et al., 2010) or does the association of the DMN with errors and mental illness indicate that such processes are simply maladaptive (Broyd et al., 2009; McVay and Kane, 2010)?

One reason why the role of the DMN in normal waking thought remains relatively mysterious may be due to the lack of an overarching framework which integrates both inter- nally and externally driven trains of thought in a unified manner (although see Burgess et al., 2007; Sonuga-Barke and Castellanos, 2007). The starting point for this review is the hypothesis that the ability to engage in an internal train of thought involves two processes: (i) spontaneous generation of mental contents (aka mental simulation) that are unrelated to direct perceptual input and arise principally from the autobiographical memory system (e.g. Schacter and Addis, 2007), and (ii) the engagement of top-down control in order to buffer and sustain this activity over time so that cognition can proceed in an orderly manner (e.g. Smallwood and Schooler, 2006). Given that the role of top-down processes in spontaneous thought is a matter of debate (e.g. McVay and Kane, 2010); Box 1 presents three potential similarities between the engagement of an internally guided train of thought and processes which are traditionally viewed as elements of top-down control.

\section{Integrating top-down control and the default mode network (DMN): a global workspace model of internally guided thought}

This paper extends a framework for spontaneous thought couched purely in information processing terms (Smallwood, 2010; Smallwood and Schooler, 2006) and is an attempt to embed the DMN in the architecture of a broad class of theories known as global workspace accounts of consciousness (Baars, 1988). Global workspace models have been presented for different attentional states (Baars et al., 2003), and tasks (Dehaene et al., 1998), and there is support for such models among the cognitive (Baars, 2002), neuroscientific (Dehaene et al., 2006), artificial intelligence (Shanahan, 2006; Shanahan and Baars, 2005) and philosophical communities (Dennett, 2001).

The neuronal global workspace theory hypothesizes that consciousness is produced through the interaction between neural submodules, which encapsulate specific sub processes (such as perception or language), and a multimodal limited capacity global workspace that allows communication between the different subsystems. This global workspace is hypothesized to involve a neural network composed of "a distributed set of cortical neurons characterized by their ability to receive from and send back to homologous neurons in other cortical areas horizontal projections through long-range excitatory axons (which may impinge on either excitatory or inhibitory neurons)" (Dehaene et al., 1998, p. 14529). Global workspace theory assumes that different mental contents (represented by the sub modules) compete for access to the workspace with conscious thought being associated with the winner. The stability of the conscious content is ensured because the global workspace has the capacity to amplify those input streams that are currently in the global workspace. 


\section{Box 1}

Three similarities between and internal train of thought and the process and functions of top-down control.

Top-down control influences how various lower level stimulusdriven processes operate and so involves operations on information that is abstract.

The engagement of an internally guided train of thought depends less upon external physical referents for cognition than does perceptually guided cognition. As a relatively greater level of abstraction is required to represent information which is independent from perceptual input (Amodio and Frith, 2006), the neural representations that are involved in internally guided thought may be more abstract than those involved in perceptually guided thought. Given that these representations themselves are of relatively high levels of abstraction, they could be capable of exerting downstream influence in a manner that may be analogous to top-down control.

Top-down control functions ensure that information flow proceeds along the lines of the current goal and does so by inhibiting irrelevant information processes.

During periods of internally guided thought, information processing is biased against the processing of perceptual information. This process of perceptual decoupling indicates that information flow within the brain is being biased in favor of relevant input (e.g. internally generated information related to the internal train of thought) rather than irrelevant input (in this case perception). This decoupling is not permanent; instead attention can be rapidly recoupled to perceptual events if salient external events occur (e.g. Smallwood et al., 2006). Nonetheless during the experience of internally generated thought, irrelevant external information is suppressed in a manner that may be analogous to the way that external distractions are suppressed during successful task performance.

Top-down control systems must have the necessary plasticity to be able to react to the changing goal demands faced by an agent.

While it is true that certain clinical disorders exhibit especially repetitive types of thought, on the whole the contents of spontaneous thought are related to the changing goal states of the individual, known as their current concerns (Klinger, 1999). For example, studies have demonstrated that the contents of consciousness are focused on upcoming personally salient events, indicating that they may play a role in the process of autobiographical planning (Smallwood et al., 2009; Morsella et al., 2010). This coupling between the current concerns of the individual and the contents of consciousness suggests that the experiences that often make up the internal train of thought are responsive to the changing situations of the individual which itself is a characteristic of top-down processes.

While generally associated with conscious perceptual experience (i.e. an externally guided train of thought), features of the global workspace architecture are also applicable to internally mediated conscious thought. A simulated neural network consisting of multiple submodules coupled to a shared pool of nodes (the shared pool representing the global workspace) showed that the access of external information into the shared pool was blocked during periods of spontaneous, non-externally triggered activity (Dehaene and Changeux, 2005). Dehaene and Changeux made two predictions on the dynamics of spontaneous thought in this particular architecture: (1) cortical areas particularly rich in 'workspace neurons' (i.e. prefrontal, parietal, superior temporal, and cingulate cortices) would exhibit the most consistent spontaneous activity during awake, conscious thought, and (2) spontaneous activity will block external stimuli from accessing the global workspace, limiting perceptual processing to the level of local sub networks. The current framework extends this basic hypothesis by suggesting that the DMN acts as an additional hub in the global workspace that may be engaged when conscious thought follows particular types of internally guided trains of thought.

Evidence suggests that this "global neuronal workspace" may rely in part upon on what has become known as the frontal-parietal network (FPN) (Corbetta et al., 2008; Vincent et al., 2008; Posner and Dehaene, 1994). Connectivity analysis derived from resting state scans (e.g. Spreng et al., 2010; Vincent et al., 2008) has defined the FPN as including the rostrolateral prefrontal cortex (rlPFC), middle frontal gyrus (mFG), anterior insula/frontal operculum(AI/FO), dorsal anterior cingulate cortex (dACC), precuneus (PCU), the caudate nucleus (CN), the dorsolateral prefrontal cortex (dLPFC) and the anterior inferior parietal lobule (aIPL). The PCU, an area with robust connections to the PCC and often observed to be active at rest, may or may not be included in the DMN due to its involvement in motor coordination and planning and other features of control (Buckner et al., 2008, see also Margulies et al., 2009). Other areas which could be involved in cognitive control include the inferior frontal gyrus (iFG) given its role in tasks requiring control (e.g. Smith and Jonides, 1999), although many consider it to be a component of the DMN. Studies of resting state connectivity suggest that the FPN shows a pattern of functional connectivity that is distinct from both the DMN and the dorsal attention network (DAN) (Spreng et al., 2010; Vincent et al., 2008). The DAN has extensive connections to the visual cortex (e.g. V1-V4) and includes the intra-parietal sulcus (IPS), frontal eye-fields (FEF), dorsal parietal cortex (DPC) and the pre-central sulcus (PCS) (Corbetta et al., 2008).

Recent discussions of the FPN suggest that it has several properties in common with the conceptualization of a global workspace hypothesized by Dehaene and colleagues. First, its anatomical location between the DMN and the DAN means the FPN "may be uniquely positioned to adjudicate between potentially competing inner- versus outer-directed processes" (Vincent et al., 2008). Second, the FPN is thought to help shift attentional focus and protect from disruption when resources are allocated to perceptual, memory, or self-referential processing (Corbetta et al., 2008). Other authors have suggested that a similar system to the FPN serves to maintain information content that is necessary for task performance on timescales of seconds (Dosenbach et al., 2008). Together, these recent views can be seen as a more neurally specified examples of the following claim associated with the functions of the global workspace: "parietal and cingulate areas contribute to the attentional gating and shifting of the focus of interest" (Dehaene and Naccache, 2001).

Fig. 1 presents a schematic of how the FPN could work in conjunction with both perceptual information from the DAN and autobiographical information provided by the DMN to selectively reinforce either internal or external trains of thought. The upper panel presents a schematic description of how perceptual and autobiographical information can be 


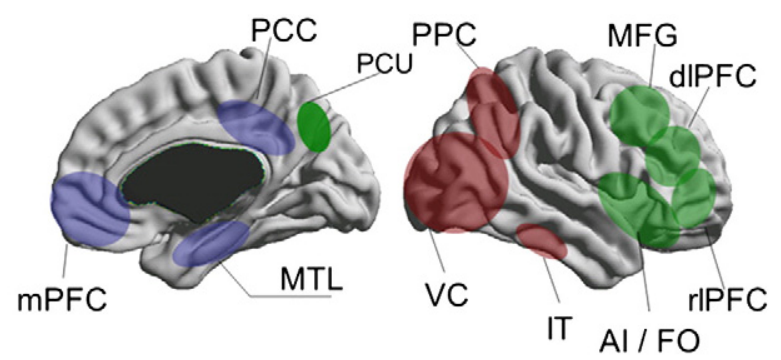

(A) Hypothetical architecture

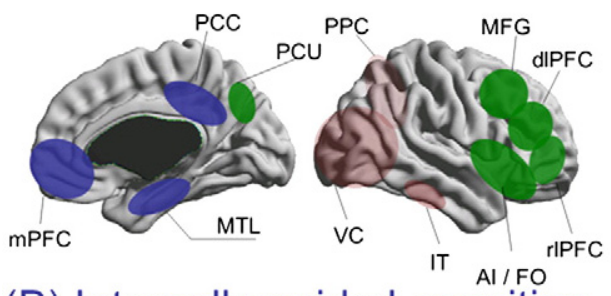

(B) Internally guided cognition

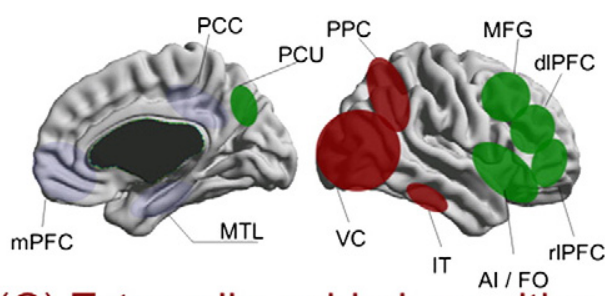

(C) Externally guided cognition

Fig. 1 - A schematized architecture outlining how internal and external trains of thought can arise in a global workspace model of the brain. The upper panel presents an example of the proposed architecture for both internally and externally generated thought. The lower left hand panel describes the neural activation which is hypothesized to occur when attention focuses on an internal train of thought. The lower right hand panel presents the neural activation that is hypothesized to occur during an external train of thought. The frontal parietal network (FPN) is represented in green, the default mode network (DMN) is represented in blue, and the dorsal attention network (DAN) in red. Note these locations areas are displayed for diagrammatic purposes only. List of regions: anterior insula/frontal operculum (AI/FO), anterior cingulate (aCC), Dorso-lateral pre-frontal cortex (dlPFC), medial Frontal Gyrus (MFG), medial Prefronal Cortex (mpFC), medial Temporal Lobe (mTL), posterior cingulate cortex (PCC), PCU Precuneus, rostro-lateral prefrontal cortex (rlPFC), Posterior Parietal Cortex (PPC) and Visual Cortex (VC).

considered as different hubs that may be mobilized into the dynamic architecture of the global workspace. The blue areas indicate brain areas involved in the DMN. The red areas indicate aspects of the visual perceptual system that are integrated with the $\mathrm{DAN}^{1}$. Areas involved in the FPN (colored green) form a 'global workspace' with the capacity to receive information from either of the submodules.

The hypothesis that the FPN and the DMN cooperate to produce the internal train of thought leads to two specific predictions (see also Dehaene and Changeux, 2005). First, situations when consciousness is internally focused should engage both the DMN and elements of the FPN. Second, periods of internally guided thought should be accompanied by a process of 'perceptual decoupling' in which sensory information from the DAN is blocked from the global workspace and so is not processed with the same detail as when the control system is required to focus on the external world. One important feature of this state of perceptual decoupling is that it may help insulate an internally maintained train of thought from being disrupted by events in the external world. The two lower panels each represent different configurations of the system that illuminate how these different predictions unfold within a simplified architecture. Each panel corresponds to different situations engaging either internally guided (B) or externally guided (C) conscious thought. When consciousness is focused internally (B), information generated

\footnotetext{
${ }^{1}$ For simplicity this schematic only focuses on visual perception but the proposal generalizes to all forms of perceptual information.
}

by the DMN gains access to the FPN, biasing attention away from concurrent visual input. In the case of an external train of thought (C), the FPN cooperates with the DAN, leading attention to be biased away from the DMN. The blocking of the DMN during states of detailed external focus helps ensure that when control is directed to the external world, externally guided cognition can proceed without disruption. In both panels, darker, opaque colors indicate amplification of the relevant submodules.

1.1. Prediction 1: conscious internal thought involves cooperation between elements of the DMN and the FPN

Initial support for a link between the DMN and internally generated thought was based on hypotheses about the psychological processes active during rest (e.g. Binder et al., 1999; Gusnard et al., 2001). In one of the earliest experiments which specifically explored the neural substrates of spontaneous thought, McGuire et al. (1997) used positron emission tomography (PET) to measure brain activity while participants performed cognitive tasks and later reported the extent of task unrelated thought (TUT) experienced; activity in a portion of the MPFC was associated with the level of TUT. Along similar lines a recent study by Andrews-Hanna et al. (2010) in which internally generated thought was measured retrospectively revealed that the MTL component of the DMN was associated with stimulus independent thoughts regarding the future or the past.

More recent studies have documented manipulations that increase both DMN activity and TUT. A study by Mason et al. 
(2007) required participants to practice visuo-spatial working memory tasks for several days. Training increased the extent of TUT (measured outside of the scanner) and activation in elements of the DMN (including aspects of the mPFC). Using a similar rationale, McKiernan et al. (2006) measured fMRI activity while varying a number of task parameters including rate of presentation and target discriminability. Both speed of presentation and task difficulty led to correlated changes in TUT reports (assessed outside of the scanner) and activity in a number of DMN structures.

Evidence that the conditions that promote activity in the DMN also increase TUT does not guarantee that both reflect the same psychological process (Gilbert et al., 2007). Evidence that activity in the DMN occurs during episodes of TUT was provided by Christoff et al. (2009) who measured TUT online during fMRI scanning. Elements of the DMN (including the mPFC and PCC) showed greater activity in periods when participants reported that they were engaged in TUT than when they were focused on the task. Fig. 2 presents a comparison of the Mason et al. and Christoff et al. studies described previously. The left hand panel shows a statistical parametric map for areas more active during practiced versus non-practiced task blocks in the Mason et al. study. The right hand panel shows areas more active during periods of TUT in the Christoff et al. study. It is apparent that similar areas of the MPFC and PCC are recruited in both nondemanding tasks (Left panel) and during transient experiences of TUT (Right panel).

A recent study by Stawarczyk et al. (2011) replicated the observation that elements of the DMN are more active during periods of TUT. One important advance in this study was the deployment of thought probes that separated the stimulus independent and task unrelated components of offtask thought. This more fine grained analysis indicated that the lateral temporal regions of the DMN were specifically related to reports of stimulus independent mentation rather than TUT. This finding suggests that one important question facing future studies of internally guided thought and/or the

(A)

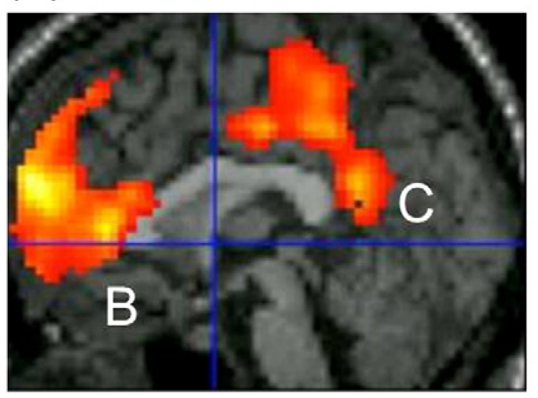

(1) Neural activation associated with internally generated thought using a blocked design (from Mason et al., 2007)
DMN is assessment of whether the DMN can be further subdivided into different constituent processes (see also Buckner et al., 2008; Andrews-Hanna et al., 2010).

The model proposed in this paper suggests that cooperation between the DMN and a system such as the FPN is necessary to produce an internal train of thought. Mason et al. observed greater precuneus activity when participants performed practiced task blocks. Further analysis indicated that this activation was most pronounced in individuals who reported frequent daydreaming. Likewise, the PCU and elements of the dorsal ACC were all active during periods of off-task thought in the data of Christoff et al. PCU activation was also observed in periods of mind wandering in the study by Stawarczyk et al. (2011). Likewise, the PCU, the IFG and elements of the dorsal ACC were all active during periods of off-task thought in the data of Christoff et al. PCU and bilateral IFG activation was also observed in periods of mind wandering in the study by Stawarczyk et al. (2011). Finally, a recent study (Dumontheil et al., 2010) demonstrated that activity in aspects of the FPN (including the rlPFC) showed a U-shaped relationship to task demands; enhanced activity was seen in both easy tasks (corresponding to the conscious processing of internally generated thought) and in difficult ones (corresponding to the conscious processing of task relevant information). Together this data from a wide range of different research groups and using several different measures of spontaneous thought support the hypothesis that circumstances that encourage TUT not only recruit the DMN, they often also engage elements of a control system such as the FPN.

If activity in the DMN and the FPN is in cooperation then they should not simply be activated at the same time; these different networks should also demonstrate functional connectivity. Initial support for this assumption was demonstrated by Smallwood et al. $(2009,2011)$ who demonstrated that engaging in a task requiring control reduced the occurrence of auto biographical planning. The strongest evidence that the

(B)

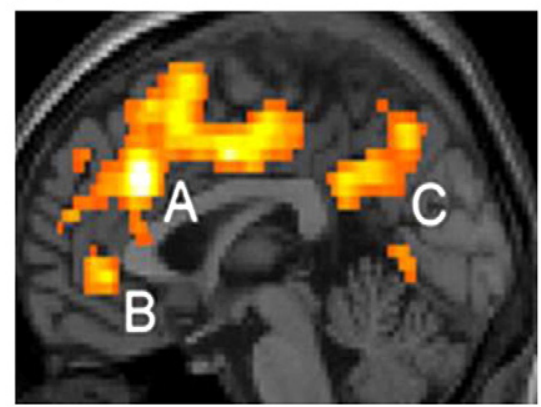

(2) Neural activation associated with internally generated thought using an event related design (from Christoff et al., 2009)

Fig. 2 - A comparison of the neural recruitment during task unrelated thought using either a blocked design (Panel A, adapted from Mason et al., 2007) or an event related design (Panel B, adapted from Christoff et al., 2009). It can be seen that in both cases activations are observed in both hubs of the default mode the medial PFC and the posterior cingulate cortex (PCC). In addition, the data from Christoff et al. reveals activations in the dorsal aCC, a part of the frontal-parietal network, providing evidence that the FPN can also be active during mind-wandering. 
FPN cooperates with the DMN to produce the internal train of thought, however, can be seen in a study of the neural processes involved in autobiographical planning (Spreng et al., 2010). Spreng et al. asked participants to either engage in a task of autobiographical planning ("How can I get out of debt?") or a visuo-spatial planning task (the Tower of London test (Shalice, 1982)). Both planning tasks recruited the FPN relative to a simple counting control. The FPN showed greater connectivity with the DAN under situations when participants engaged in visuo-spatial planning. Critically, however, connectivity analysis indicated that elements of the DMN showed enhanced connectivity with elements of the FPN when participants engaged in conscious autobiographical planning. The data of Spreng and colleagues indicates autobiographical planning depends upon cooperation between the FPN and the $\mathrm{DMN}$; these processes are also routinely found to compose the content of TUT (Smallwood et al., 2009; Smallwood et al., 2011; Smallwood and O'Connor, in press). The observation that autobiographical planning occupies a significant proportion of the internal train of thought and depends upon the FPN provides strong construct validity for the claim that spontaneous task unrelated experiences can be implicated in the process of executive control (e.g. Smallwood, 2010).

\subsection{Prediction 2: engaging the internal stream of thought dampens sensory processing}

One of the most common metaphors for attention is that of a spotlight which amplifies relevant over irrelevant input (Posner and Petersen, 1990; Posner and Dehaene, 1994). While usually focused on different modalities of perception (such as auditory versus visual), the current framework assumes that the spotlight of attention can also focus internally. A focus of attention on an internal input stream necessarily denies amplification to other input streams (in this case all perceptual information, e.g. Ray and Coles, 1985) leading to an association between internal thought and the state of perceptual decoupling (Smallwood et al., 2003). This is predicted by the global workspace architecture because according to that framework spontaneous activity and external input streams are in competition for the same shared resource (such as the FPN) (Dehaene and Changeux, 2005).

An important feature of the process of perceptual decoupling is that it allows the consideration of information that is not present in the immediate environment and instead is derived largely from memory (e.g. imaginative thought). Decoupling frees consciousness from the constraints of attending to the here and now, allowing the agent the chance to "avoid the world of actions and sensations, and stay in the subjective world of desires and feelings" (Frith, 2007, p. 105). One of the many situations where decoupling serves a necessary function is in mental state attribution. One such example is the "Sally Ann" task used in assessing theory of mind in children (Wimmer and Perner, 1983), in which successful performance requires a focus on mental representations that are at odds with current physical reality (where Sally thinks the marble is, rather than where it is now). Meta-analysis suggests that the decoupling involved in theory of mind is similar to that which occurs during internally guided thought; both recruit similar neural substrates involving elements of the DMN (Gallagher and Frith, 2003;

\section{Box 2}

Three reasons why internally guided thought involves a state of perceptual decoupling.

Reason 1: Internally guided thought does not depend directly on perception.

The content of an internal train of thought is the product of the autobiographical system (e.g. Schacter and Addis, 2007). This reliance of internal trains of thought on memorial content means that concurrent perceptual information has less direct relevance than if cognition is focused on an external event. Given that internally derived thought can proceed without perceptual input, there is no principled reason to expect sensory information to be processed in any degree of detail.

Reason 2: Conscious experience has limited capacity.

One notable feature of conscious experience is that it is serial; it becomes diminished when attention is divided between many items or tasks. One implication of this relatively limited capacity is that engagement in one input stream requires that other streams be neglected. Once it is recognized that consciousness can focus on an internally generated input stream in a detailed manner, it follows that other input streams (in this case perception) would be neglected.

Reason 3: Internally generated thought must be buffered from the distractions of the external environment

Often in psychological experiments irrelevant distracting information is a feature of the external task (e.g. the incongruent color information in the Stroop task, (Stroop, 2004)). When engaged in a daydream, however, perceptual events are irrelevant to the specific train of thought that is being followed. To an agent attempting to daydream, the success with which an internally generated train of thought is maintained depends in part upon the extent to which the distracting input of the external environment is suppressed.

Spreng, Mar and Kim, 2008, see also Spreng and Grady, 2010). Box 2 summarizes three reasons why an internal train of thought often involves a state of perceptual decoupling.

There is now abundant evidence that attention is decoupled from perception during the engagement of an internal train of thought. For example, the occurrence of TUT during memory tasks reduces a participant's ability to recall information that was concurrently presented. An example of this absent minded forgetting is presented in Fig. 3 (Upper right panel) where it can be seen that word recall was significantly less accurate when those words were encoded during periods of TUT (Smallwood et al., 2003). Similarly, reading comprehension is impaired when the participant drifts off task and engages in mindless reading (see Smallwood, 2011 for a more detailed review). Fig. 3 (Upper left panel) illustrates this effect in the context of reading a detective novel. Participants who engaged in TUT during periods when the author revealed a fact relevant to solving a crime (red bar) were less likely to solve the crime than those who were on task when reading the clues (blue bar, Smallwood et al., 2008c). Other studies demonstrated abnormal reading times associated with such mindless reading during narrative comprehension (Raichle et al., 2001) coupled with a greater blinking rate (Smilek et al., 2010a,b). The effects of decoupling are also apparent in other tasks. TUT leads to errors during sustained 


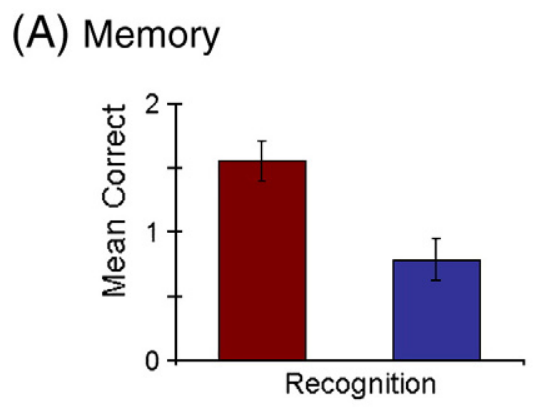

(C) Task Representations

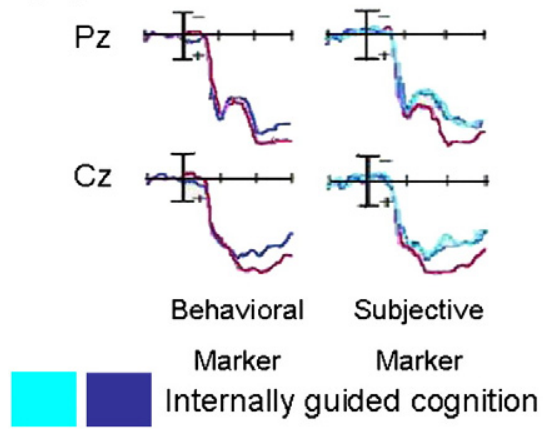

(B) Textual Inference

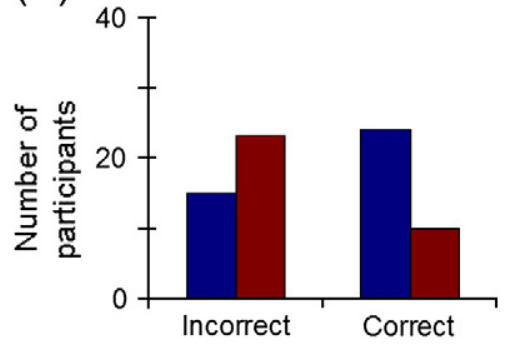

(D) Sensory Information

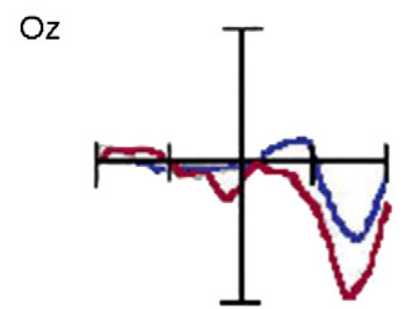

Externally guided cognition

Fig. 3 - Examples of behavioral and subjective indicators of perceptual decoupling during internally generated thought. The upper panels present behavioral consequences of decoupling in two common situations for studying internal thought: (A) during TUT encoding suffers from absent-minded forgetting (adapted from Smallwood et al., 2007) and (B) while reading TUT leads to mindless reading (adapted from Smallwood et al., 2008a). The lower panel presents event-related potential evidence for decoupling based on the extent to which task relevant information is suppressed by TUT. Both task representations as indexed by the amplitude of the P3 (adapted from Smallwood et al., 2008b) and sensory ERPs (adapted from Kam et al., 2010) are reduced during periods of TUT.

attention (e.g. Smilek et al., 2010a,b; McVay and Kane, 2009; Smallwood et al., 2004; Smallwood et al., 2007) and compromises the generation of random numbers (Teasdale et al., 1995).

EEG studies provide a sensitive indicator of perceptual decoupling because the examination of the magnitude of event related potentials (ERPs) allow the level of information processing of external perceptual events to be quantified. ERPs are calculated by averaging the brain's response to external events that are processed during a period of task performance. By comparing the magnitude of the ERPs during periods of externally and internally guided thought it is possible to explicitly test the idea that perceptual decoupling occurs when attention is directed internally. As can be seen in the lower left panel of Fig. 3, the P3 event related potential (ERP) to a target, recorded at the midline central and parietal electrodes, showed reduced amplitude during periods of TUT relative to periods of task focus (Smallwood et al., 2008c). In a more recent study (Kam et al., 2010) sensory processing was measured by the cortical response to task-irrelevant events (such as a visual grating or an auditory beep). An example of the data from Kam and colleagues is presented in the lower left panel of Fig. 3, which shows the task-irrelevant sensory ERP recorded at a central electrode over the occipital cortex. In this panel, it can be seen that the amplitude of the visual response occurring in the first $100 \mathrm{~ms}$ after the event is larger during periods of task focus (red) than during TUT (blue). The same study showed that auditory processing (measured by N1 amplitude) was also reduced during TUT. Other studies have documented that TUT reduces the processing of both task relevant and distracter events in a task (Barron et al., 2011) indicating that the state of decoupling reduces external attention and yet can be distinct from a state of distractibility. Finally, the perceptual decoupling that occurs when the mind wanders interferes with the processing of external events even when there is no external task at all (Brabosczcz and Delorme, 2010). Taken together this growing body of evidence using ERPs confirms a key tenet of the decoupling hypothesis: detailed internal mentation that is at odds with perceptual input dampens the processing of external information.

Parallel evidence for perceptual decoupling comes from studies of DMN functional connectivity and investigations of the neural precursors to error. Studies indicate decreased activation in several areas of the sensory cortices occurs during periods when the DMN is active. Following the observations of Greicius and Menon (2004) this has led to the notion of anticorrelation between the DMN and the taskpositive DAN network (e.g. Fox et al., 2005; Fransson, 2005; Vincent et al., 2008). This anticorrelation can also interfere with the performance of demanding tasks. A growing number of studies have documented that the DMN is especially active during periods prior to errors or lengthy response times on tasks that depend upon a detailed cognitive analysis (e.g. Eichele et al., 2008; Christoff et al., 2009; Weissman et al., 2006) and leads to absent minded forgetting (e.g. Kim, 2010). These 
observed anticorrelations between the DMN and sensory systems, along with the association of DMN activity with task errors, not only parallels the results of the study of TUT but also provides further support for the notion that the engagement of a detailed internal train of thought is accompanied by an absence of a detailed focus on the external world (i.e. perceptual decoupling). Importantly, this mutual inhibition between perception and internal thought would emerge naturally within an architecture such as that described in this paper in which both input streams compete for access to a shared pool of resources (such as the global workspace).

\subsection{Future directions}

In order to escape from the constraints placed on it by the environment, the mind must be able to both generate spontaneous mental contents which are unrelated to perception and coordinate these experiences so that they are maintained in the face of competing sensory information. The current account proposes that the internal train of thought is produced when the DMN (providing the autobiographical content) cooperates with a control network (such as the FPN) which helps in either the coordination of these thoughts themselves or by buffering this internal stream against disruption by perception. This cooperation explains why the neural recruitment associated with spontaneous thought engages elements of both the DMN and FPN systems. Moreover, assigning to the FPN at least part of the function of the "global workspace" explains why spontaneous thought and perceptual decoupling co-occur; perceptual and spontaneous information compete for limited workspace resources.

While the data reviewed are consistent with the proposed hypothesis, research which specifically focuses on both the generation of the internal train of thought and the implications of this state for ongoing perception is still scarce and further work is needed before firm conclusions can be drawn. Moreover, the present framework is intentionally general and therefore leaves many important questions unanswered (see Box 3). One overarching question, given the recognized contribution of elements of the DMN (such as the mPFC) to processes such as mental state attribution (e.g. Gallagher and Frith, 2003; Mitchell, 2010) is whether cooperation between the DMN and the FPN may also support the capacity to sustain a detailed simulation of the goals and desires of other people.

Notwithstanding such limitations, the current hypothesis provides a framework which can usefully constrain future research. According to the current proposal, for example, situations that prohibit the opportunity for a detailed internal train of thought may do so because of involvement of the FPN in the external task. In such an experiment the FPN may well be equally engaged in both internally and externally guided thought but is critically focused on different input streams; this can be seen most clearly in the data of Spreng et al. (2010). In this context, comparisons between conditions will illuminate the neural processes that differ (e.g. the involvement of the DMN) but will obscure any similarities (because the FPN is engaged in either the external task or the internal train of thought). By contrast, the use of experience sampling probes online during fMRI scanning allows the comparison of situations when participants' attend to the performance of a

\section{Box 3}

Outstanding questions on the neuro-cognitive basis of the internal train of thought.

Do all varieties of internally generated conscious thoughts engage the same neural substrates?

There are likely to be many different types of internally generated trains of thought that individuals can engage in. One limitation of the current account is that it may be most applicable to those processes that are employed in a specific type of autobiographical planning. Although a common form of spontaneous thought, other varieties of thought could engage different neural substrates, such as those involved in person perception (e.g. Mason and Macrae, 2004; Hoffman and Haxby, 2000), emotion (Adolphs, 2002), or empathy (Singer et al., 2004).

What is the significance of activity in the DMN when attention is not engaged in an internal train of thought?

Studies indicate that DMN activity can continue (albeit in an altered manner) in situations in which the opportunity for conscious internal thought is substantially reduced (Esposito et al., 2006). Whether unattended information is preconscious or unconscious can be addressed in a straightforward manner for perception, but novel methods will be needed to experimentally assess how to categorize DMN activity which is not globally available.

What is the fate of external events that are presented during perceptual decoupling?

While explicit recollection is impaired, certain features of stimulus processing may be preserved, raising the possibility that external events could indirectly prime the contents of the internal train of thought. Recently, Miles and colleagues demonstrated that the temporal focus of mind-wandering is influenced by the direction of task-irrelevant apparent motion (Miles et al., 2010). Identifying whether external events can lead to priming during TUT is a important question for future research.

What is the time course of internally generated thought?

Studies of the low frequency fluctuations in the DMN (Sonuga-Barke and Castellanos, 2007) and behavioral precursors of attentional lapses (Eichele et al., 2008) indicate that internally generated thoughts are associated with changes on the timescale of seconds and so are distinct from much shorter lapses such as the attentional blink (Shapiro et al., 1997).

Why does the mind wander?

The current framework focuses on how an internal train of thought is produced; it does not explain why the mind wanders. Within the literature there are three proposed explanations for why these shifts occur: 1) mind-wandering occurs because suppressed mental contents become more accessible (Wegner, 2009), 2) mind-wandering may occur because of an intermittent failure to re-represent the contents of consciousness (known as meta-awareness. (Schooler, 2002)) and 3) lapses in 'control' allow the mind to escape from the constraints of what it is doing (McVay and Kane, 2010). Further research is needed to assess whether in isolation or in combination these different explanations can account for the emerging cognitive neuroscience data on spontaneous thought.

simple task (which may not recruit the FPN) with periods of internally focused cognition (which we argue should involve the FPN). This is one possible reason why studies that used 
thought sampling observed recruitment in areas associated with control, e.g. the dLPFC and ACC (Christoff et al., 2009) and those that have been implicated in both DMN and control (such as the IFG, Christoff et al., 2009; Stawarczyk et al., 2011). By contrast, studies which compared situations that do and do not allow an internal train of thought to occur, more often than not observe activation in the DMN (e.g. Mason et al., 2007) $)^{2}$.

Perhaps the most important implication of the current hypothesis is that it offers an explanation for the anticorrelation between DMN activity and perception. Assuming that the global workspace can only accommodate a subset of current input streams incompatible input streams (such as mulling over what I will do tomorrow versus paying attention to what I am doing now) could not both be processed in great detail. As global availability 'amplifies' relevant streams over irrelevant streams, the process of competition combined with subsystem inhibition would ensure that fundamentally incompatible input streams are anticorrelated over time ${ }^{3}$. In the proposed architecture, therefore, the anticorrelation between the DMN and the DAN emerges naturally from the competition between different input streams for the limited capacity of the global workspace architecture (e.g. the FPN). Given that the stimulus-independent nature of DMN activity is its most intriguing feature, understanding whether the accompanying sensory neglect arises in part due to interaction with the global workspace will constrain how the DMN's role in waking thought is understood.

\section{Acknowledgments}

Thanks to Boris Bernhardt for his help with Fig. 1 and to Haakon Engen and Florence Ruby for their comments on the arguments presented in this paper.

\section{REFERENCES}

Adolphs, R., 2002. Neural systems for recognizing emotion. Current Opinion in Neurobiology 12 (2), 169-177.

\footnotetext{
${ }^{2}$ It is important to note that this limitation also applies to the comparison of correct and incorrect task responses. According to the current framework, an internal train of thought would be most associated with task errors in circumstances when correct performance requires elements of the FPN. However, this contrast is between correct responses (which should involve the FPN) and incorrect responses (some of which involve FPN-supported TUT). Comparing correct and incorrect performance will tend to obscure any activation in the FPN that is involved in an internal train of thought while emphasizing the differences (e.g. DMN activation).

${ }^{3}$ It is important to note that it is the incompatibility between the input streams that determines the competition for access to the global workspace. In many situations (such as self reference, for a review see Mitchell, 2009) elements of the DMN are directed to the processing of incoming perceptual events. These situations suggest that it maybe the incompatibility between input streams (rather than activity in the DMN per se) that cause competition with global workspace.
}

Amodio, D.M., Frith, C.D., 2006. Meeting of minds: the medial frontal cortex and social cognition. Nature Reviews. Neuroscience 7 (4), 268-277.

Andrews-Hanna, J., Reidler, J.S., Huang, C., Poulin, R., Buckner, R.L., 2010. Evidence for the default network's role in spontaneous cognition. Journal of Neurophysiology 104, 322-305.

Baars, B.J., 1988. A Cognitive Theory of Consciousness. Cambridge Univ Pr.

Baars, B.J., 2002. The conscious access hypothesis: origins and recent evidence. Trends in Cognitive Sciences 6 (1), 47-52.

Baars, B.J., Ramsoy, T.Z., Laureys, S., 2003. Brain, conscious experience and the observing self. Trends in Neurosciences 26 (12), 671-675.

Barron, E., Riby, L., Greer, J., Smallwood, J., 2011. Absorbed in Thought. Psychological Science.

Binder, J.R., Frost, J.A., Hammeke, T.A., Bellgowan, P.S.F., Rao, S.M., Cox, R.W., 1999. Conceptual processing during the conscious resting state: a functional MRI study. Journal of Cognitive Neuroscience 11 (1), 80-93.

Brabosczcz, C., Delorme, A., 2010. Lost in thoughts: neural markers of low alertness during mind wandering 4, 14 NeuroImage 54, 3040-3047.

Broyd, S.J., Demanuele, C., Debener, S., Helps, S.K., James, C.J., Sonuga-Barke, E.J.S., 2009. Default-mode brain dysfunction in mental disorders: a systematic review. Neuroscience and Biobehavioral Reviews 33 (3), 279-296.

Buckner, R.L., Andrews-Hanna, J.R., Schacter, D.L., 2008. The brain's default network. Annals of the New York Academy of Sciences 1124 (1), 1-38.

Burgess, P.W., Dumontheil, I., Gilbert, S.J., 2007. The gateway hypothesis of rostral prefrontal cortex (area 10) function. Trends in Cognitive Sciences 11 (7), 290-298.

Christoff, K., Gordon, A.M., Smallwood, J., Smith, R., Schooler, J.W., 2009. Experience sampling during fMRI reveals default network and executive system contributions to mind wandering. Proceedings of the National Academy of Sciences 106 (21), 8719.

Corbetta, M., Shulman, G.L., 2002. Control of goal-directed and stimulus-driven attention in the brain. Nature Reviews. Neuroscience 3 (3), 201-215.

Corbetta, M., Patel, G., Shulman, G.L., 2008. The reorienting system of the human brain: from environment to theory of mind. Neuron 58 (3), 306-324.

Dehaene, S., Changeux, J.P., 2005. Ongoing spontaneous activity controls access to consciousness: a neuronal model for inattentional blindness. PLoS Biology 3 (5), e141.

Dehaene, S., Kerszberg, M., Changeux, J.P., 1998. A neuronal model of a global workspace in effortful cognitive tasks. Proceedings of the National Academy of Sciences of the United States of America 95 (24), 14529.

Dehaene, S., Changeux, J.P., Naccache, L., Sackur, J., Sergent, C., 2006. Conscious, preconscious, and subliminal processing: a testable taxonomy. Trends in Cognitive Sciences 10 (5), 204-211.

Dehaene, S., Naccache, L., 2001. Towards a cognitive neuroscience of consciousness: basic evidence and a workspace framework. Cognition 79, 1-37.

Dennett, D., 2001. Are we explaining consciousness yet? Cognition 79 (1-2), 221-237.

Dosenbach, N.U.F., Fair, D.A., Cohen, A.L., Schaggar, B.L., Petersen, S.L., 2008. A dual-networks architecture of top down control. Trends in Cognitive Sciences 12 (3), 99-105.

Dumontheil, I., Gilbert, S.J., Frith, C.D., Burgess, P.W., 2010. Recruitment of lateral rostral prefrontal cortex in spontaneous and task-related thoughts. The Quarterly Journal of Experimental Psychology 63 (9), 1740-1756.

Eichele, T., Debener, S., Calhoun, V.D., Specht, K., Engel, A.K., Hugdahl, K., Von Cramon, D.Y., et al., 2008. Prediction of human errors by maladaptive changes in event-related brain networks. Proceedings of the National Academy of Sciences 105 (16), 6173. 
Esposito, F., Bertolino, A., Scarabino, T., Latorre, V., Blasi, G., Popolizio, T., Tedeschi, G., et al., 2006. Independent component model of the default-mode brain function: assessing the impact of active thinking. Brain Research Bulletin 70 (4-6), 263-269.

Fox, M.D., Snyder, A.Z., Vincent, J.L., Corbetta, M., Van Essen, D.C., Raichle, M.E., 2005. The human brain is intrinsically organized into dynamic, anticorrelated functional networks. Proceedings of the National Academy of Sciences of the United States of America 102 (27), 9673.

Fransson, P., 2005. Spontaneous low-frequency BOLD signal fluctuations: an fMRI investigation of the resting-state default mode of brain function hypothesis. Human Brain Mapping 26 (1), 15-29.

Frith, C., 2007. Making up the Mind. Blackwell, London.

Gallagher, H.L., Frith, C.D., 2003. Functional imaging of theory of mind. Trends in Cognitive Sciences 7 (91-96).

Gilbert, S.J., Dumontheil, I., Simons, J.S., Frith, C.D., Burgess, P.W., 2007. Comment on "Wandering minds: the default network and stimulus-independent thought". Science 317 (5834), 43.

Greicius, M.D., Menon, V., 2004. Default-mode activity during a passive sensory task: uncoupled from deactivation but impacting activation. Journal of Cognitive Neuroscience 16 (9), 1484-1492.

Gusnard, D.A., Akbudak, E., Shulman, G.L., Raichle, M.E., 2001. Medial prefrontal cortex and self-referential mental activity: relation to a default mode of brain function. Proceedings of the National Academy of Sciences 98 (7), 4259.

Hoffman, E.A., Haxby, J.V., 2000. Distinct representations of eye gaze and identity in the distributed human neural system for face perception. Nature Neuroscience 3 (1), 80-84.

James, W., 1890. The Principles of Psychology. Henry Holt, New York. Volume 1.

Kam, J.W., Dao, E., Farley, J., Fitzpatrick, K., Smallwood, J., Schooler, J.W., Handy, T.C., 2010. Slow fluctuations in attentional control of sensory cortex (Early Access) Journal of Cognitive Neuroscience 1-11.

Kane, M.J., Brow, L.H., McVay, J.C., Sylvia, P.J., Myin-Germeys, I., Kwapil, T.R., 2007. For whom the mind wanders, and when. Psychological Science 18, 614-621.

Kim, H., 2010. Neural activity that predicts subsequent memory and forgetting: a meta-analysis of $74 \mathrm{fMRI}$ studies. NeuroImage 50 (4), 1648-1657.

Klinger, E.C., 1999. Thought flow: properties and mechanisms underlying shifts in content. In: Singer, J.A., Salovey, P. (Eds.), At play in the fields of consciousness: Essays in the honour of Jerome L. Singer. Lawrence Erlbaum Associates, Inc, Mahwah, NJ 29-50.

Margulies, D.S., Vincent, J.L., Kelly, C., Lohmann, G., Uddin, L.Q., Biswal, B.S., Villinger, A., Castellanos, F.X., Milham, M.P., Petrides, M., 2009. Precuenus shares intrinsic functional architecture in humans and monkeys. Proceedings of the National Academy of Sciences 106 (47), 20069-20074.

Mason, M.F., Macrae, C.N., 2004. Categorizing and individuating others: the neural substrates of person perception. Journal of Cognitive Neuroscience 16 (10), 1785-1795.

Mason, M.F., Norton, M.I., Van Horn, J.D., Wegner, D.M., Grafton, S.T., Macrae, C.N., 2007. Wandering minds: the default network and stimulus-independent thought. Science 315 (5810), 393-395.

McGuire, P., Paulesu, E., Frackowiak, R., Frith, C., 1997. Brain activity during stimulus independent thought. Schizophrenia Research 24 (1-2), 169.

McKiernan, K.A., D’Angelo, B.R., Kaufman, J.N., Binder, J.R., 2006. Interrupting the "stream of consciousness": an fMRI investigation. NeuroImage 29 (4), 1185-1191.

McVay, J.C., Kane, M.J., 2009. Conducting the train of thought: working memory capacity, goal neglect, and mind wandering in an executive-control task. Journal of Experimental Psychology: Learning 196-204.
McVay, J.C., Kane, M.J., 2010. Does mind wandering reflect executive function or executive failure? Comment on Smallwood and Schooler (2006) and Watkins (2008) Psychological Bulletin 136 (2), 188.

Miles, L.K., Karpinska, K., Lumsden, J., Macrae, C.N., 2010. The meandering mind: vection and mental time travel. Plos One 5 (5), e10825.

Mitchell, J., 2010. Social psychology as a natural kind. Trends in Cognitive Science 13 (6), 246-251.

Morsella, E., Ben-Zeev, A., Lanska, M., Bargh, J.A., 2010. The spontaneous thoughts of the night: how future tasks breed intrusive cognitions. Social Cognition 28 (5), 641-650.

Posner, M.I., Dehaene, S., 1994. Attentional networks. Trends in Neurosciences 17 (2), 75-79.

Posner, M.I., Petersen, S.E., 1990. The attention system of the human brain. Annual Review of Neuroscience 13 (1), 25-42.

Raichle, M.E., 2009. A paradigm shift in functional brain imaging. The Journal of Neuroscience 29 (41), 12729.

Raichle, M.E., Snyder, A.Z., 2007. A default mode of brain function: a brief history of an evolving idea. NeuroImage 37 (4), 1083-1090.

Raichle, M.E., MacLeod, A.M., Snyder, A.Z., Powers, W.J., Gusnard, D.A., Shulman, G.L., 2001. A default mode of brain function. Proceedings of the National Academy of Sciences of the United States of America 98 (2), 676-682.

Ray, W.J., Cole, H.W., 1985. EEG alpha activity reflects attentional demands, and beta activity reflects emotional and cognitive processes. Science 228 (4700), 750.

Schacter, D.L., Addis, D.R., 2007. Constructive memory: the ghosts of past and future. Nature 445 (7123), 27.

Schooler, J.W., 2002. Re-representing consciousness: dissociations between experience and meta-consciousness. Trends in Cognitive Sciences 6 (8), 339-344.

Shalice, T., 1982. Specific impairments of planning. Philosophical Transactions of the Royal Society of London B 298, 199-209.

Shanahan, M., 2006. A cognitive architecture that combines internal simulation with a global workspace. Consciousness and Cognition 15 (2), 433-449.

Shanahan, M., Baars, B., 2005. Applying global workspace theory to the frame problem. Cognition 98 (2), 157-176.

Shapiro, K.L., Raymond, J.E., Arnell, K.M., 1997. The attentional blink. Trends in Cognitive Sciences 1 (8), 291-296.

Singer, T., Seymour, B., O’Doherty, J., Kaube, H., Dolan, R.J., Frith, C.D., 2004. Empathy for pain involves the affective but not sensory components of pain. Science 303 (5661), 1157.

Smallwood, J., 2010. Why the global availability of mind wandering necessitates resource competition: reply to McVay and Kane (2010). Psychological Bulletin 136 (2), 202.

Smallwood, J., 2011. Mind-wandering while reading: mindless reading, attentional decoupling and the cascade model of inattention. Language and Linguistic Compass 5 (2), 63-77.

Smallwood, J., \& O'Connor, R.C., (in press). Imprisoned by the past: unhappy moods encourage a retrospective focus to mind wandering. Cognition \& Emotion.

Smallwood, J., Schooler, J.W., 2006. The restless mind. Psychological Bulletin 132 (6), 946-958.

Smallwood, J.M., Baracaia, S.F., Lowe, M., Obonsawin, M., 2003. Task unrelated thought whilst encoding information. Consciousness and Cognition 12 (3), 452-484.

Smallwood, J., Davies, J.B., Heim, D., Finnigan, F., Sudberry, M., O'Connor, R., Obonsawin, M., 2004. Subjective experience and the attentional lapse: task engagement and disengagement during sustained attention. Consciousness and Cognition 13 (4), 657-690.

Smallwood, J., Riby, L., Heim, D., Davis, J.B., 2006. Encoding during the attentional lapse. Consciousness and Cognition 15 (1), 218-231.

Smallwood, J., McSpadden, M., Schooler, J.W., 2007. The lights are on but no one's home: meta-awareness and the decoupling of 
attention when the mind wanders. Psychonomic Bulletin \& Review 14 (3), 527.

Smallwood, J., Beech, E.M., schooler, J.W., Handy, T.C., 2008a. Going AwOL in the brain - mind wandering reduces the cortical analysis of the task environment. Journal of Cognitive Neuroscience 20 (3), 458-469.

Smallwood, J., McSpadden, M.C., Schooler, J.W., 2008b. When attention matters: the curious incident of the wandering mind. Memory \& Cognition 36 (6), 1144-1150.

Smallwood, J., McSpadden, M., Luus, B., Schooler, J., 2008c. Segmenting the stream of consciousness: the psychological correlates of temporal structures in the time series data of a continuous performance task. Brain and Cognition 66 (1), 50-56.

Smallwood, J., Nind, L., O'Connor, R.C., 2009. When is your head at? An exploration of the factors associated with the temporal focus of the wandering mind. Consciousness and Cognition 18 (1), 118-125.

Smallwood, J., Schooler, J.W., Turk, D.J., Cunnigham, S.J., Burns, P., Macrae, C.N., 2011. Self reflection and the temporal focus of the wandering mind. Consciousness and Cognition.

Smilek, D., Carriere, J., Cheyne, J., 2010a. Out of mind, out of sight: eye blinking as indicator and embodiment of mind wandering. Psychological Science 21 (6), 786-789 January 01.

Smilek, D., Carriere, J.S., Cheyne, J.A., 2010b. Failures of sustained attention in life, lab, and brain: ecological validity of the SART. Neuropsychologia 48 (9), 2564-2570.

Smith, E.E., Jonides, J., 1999. Storage and Executive Processes in the Frontal Lobes. Science 283 (5408), 1657-1661.

Sonuga-Barke, E.J.S., Castellanos, F.X., 2007. Spontaneous attentional fluctuations in impaired states and pathological conditions: a neurobiological hypothesis. Neuroscience and Biobehavioral Reviews 31 (7), 977-986.
Spreng, R., Grady, C., 2010. Patterns of brain activation supporting autobiographical memory, prospection, and theory of mind. Journal of Cognitive Neuroscience 21, 489-510.

Spreng, R., Mar, R., Kim, A., 2008. The common neural basis of autobiographical memory, prospection, navigation, theory of mind and the default mode: a quantitative meta-analysis. Journal of Cognitive Neuroscience 21 (3), 489-510.

Spreng, R.N., Stevens, W.D., Chamberlain, J.P., Gilmore, A.W., Schacter, D.L., 2010. Default network activity, coupled with the frontoparietal control network, supports goal-directed cognition. NeuroImage 53 (1), 303-317.

Stawarczyk, D., Majerus, S., Maquet, P., Argembeau, A., 2011. Neural correlates of ongoing conscious experience: both task unrelatedness and stimulus independence are related to default network activity. PloS One 6 (2), e16997 10.371/ journalpone0016997.

Stroop, J.R., 2004. Studies of interference in serial verbal reactions. Cognitive Psychology: Key Readings 193.

Teasdale, J.D., Dritschel, B.H., Taylor, M.J., Proctor, L., Lloyd, C.A., Nimmo-Smith, I., Baddeley, A.D., 1995. Stimulus-independent thought depends on central executive resources. Memory \& Cognition 23, 551-551.

Vincent, J.L., Kahn, I., Snyder, A.Z., Raichle, M.E., Buckner, R.L., 2008. Evidence for a frontoparietal control system revealed by intrinsic functional connectivity. Journal of Neurophysiology 100 (6), 3328.

Wegner, D.M., 2009. How to think, say, or do precisely the worst thing for any occasion. Science 325 (5936), 48.

Weissman, D.H., Roberts, K.C., Visscher, K.M., Woldorff, M.G., 2006. The neural bases of momentary lapses in attention. Nature Neuroscience 9 (7), 971-978.

Wimmer, H., Perner, J., 1983. Beliefs about beliefs: representation and constraining function of wrong beliefs in young children's understanding of deception. Cognition 13 (1), 103-128. 